\title{
The preference of rats for a more difficult task'
}

HERBERT FRIEDMAN, ROGER M. TARPY, 2 and PETER KOMELSKI, College of William and Mary, Williansburg, Va. 23185

Twelve rats were trained in a Y-maze with a hurdle (requiring high effort) in the right arm. Two food rewards, equal in weight and acceptability but discriminably different. were used. All choices were rewarded with one of the two types of reward. The group given each reward randomly preferred the nonhurdle side. Groups given different rew'ards for each arm preferred the hurdle side. These results support Premack's theory of reinforcement.

It has generally been assumed that requiring greater effort for a response reduces the tendency to repeat that response (Hull, 1943). Greater effort has been shown to decrease resistance to extinction (Mowrer \& Jones, 1945), although the opposite effect has been reported (Lawrence \& Festinger, 1962; Maatsch, Adelman, \& Denny, 1954). There are recent reports of no effect of effort on rate of responding (Chung, 1965) and resistance to extinction (Stachnik, 1967). Lewis (1964) and Aaronson (1961) have shown that effort can increase the value of the reward object.

The present experiment was a direct test of the effect of response effort upon the value of a reward. Two food rewards, similar in preferability but discriminably different, were used to facilitate the conditioning of differential cue or secondary reward value. All Ss were trained under both effort conditions, and subsequent preference for the difficult response was measured.

\section{Subjects and Apparatus}

\section{METHOD}

The Ss were 12 male albino rats. They were trained in a gray, symmetrical Y-maze which was covered to reduce extraneous cues. The arms of the maze were $30 \times 4 \times 6$ in. A box $12 \mathrm{in.}$ long at the end of each arm served as the goal box as well as the start box for the next trial; thus, the Ss could receive training without intertrial handling. On all trials, the right arm relative to the particular start box contained a hurdle placed just beyond the choice point. This hurdle was a black Masonite board, 18 in. long, on which five unequally spaced $3 / 4$-in. barriers $\left(1 \frac{1 / 2}{2}\right.$ in. high) were placed extending the width of the arm.

Procedure

Pretraining consisted of seven blocks of 30 trials, one block on the first day and two on each successive day, spaced $1 / 2 h$ apart. The average intertrial interval was $30 \mathrm{sec}$. These trials were forced choice with half the trials in each block to each side. Following were 14 blocks (two blocks/day) of free choice responding in which Ss cou'd choose either the hurdle (right) or the easy (left) arm.

Reinforcement procedures were consistent for any given $\mathrm{S}$ throughout training. Each response was rewarded with a single pellet of one of two types of food-Noyes pellets or Kix dry cereal (General Mills) which were of the same average weight (45 $\mathrm{mg}$ ) and initial acceptability but markedly different in size, shape, texture, and taste. On the first six blocks of free choice trials, the control group (RR) was randomly reinforced with one pellet of either type on all trials. The rewards for the other groups, however, were correlated with the arms. Group CR-K always received Kix for the hurdle side and Noyes pellets for the easy arm. Group CR-N received Noyes pellets at the hurdle side and $\mathrm{Kix}$ at the easy arm. On the remaining eight blocks, Ss were given training with reward conditions reversed so that the $R R$ group received reinforcement correlated with difficulty while the $C R$ groups were given random reinforcement.

The performance during free-choice trials is shown in Fig. 1. All Ss in the randomly reinforced (RR) group preferred the left (easy) arm while the Ss in the correlated reinforcement (CR) groups ranged from no preference to $100 \%$ choice of the hurdle arm, though in terms of reward CR-K chose Kix, which CR-N avoided. The difference in preference between the RR and CR Ss was highly significant ( $p<.002$, U-test). The mean preferences increased during free-choice experience to a final level of about $84 \%$ of the trials to the easy arm (no hurdle) for Group RR and $76 \%$ and $70 \%$ to the hurdle arm for CR-K and CR-N. Total trials of Block 6 were significantly to the left side $(p<.001)$ for Group RR and to the hurdle side $(\mathrm{p}<.001)$ for the CR groups (binomial test, Siegel, 1956). Reversing conditions for Group RR, correlating the reinforcements with the arms, resulted in an increased tendency to choose the hurdle arm by all Ss with eight blocks of continued free-choice trials. For the CR Ss the preference for the hurdle arm was difficult to eliminate or reduce by using random reinforcement.

DISCUSSION

Since all groups initially had the same experience with regard to number of trials to each side and the number of each type of reward received, differences among the groups in free-choice preference must be attributed to the manner of association of each type of reward with each arm.

The assumption tested in the experiment was that the reward properties associated with the more difficult response are effectively enhanced. Under the CR conditions the enhanced reward could be obtained only by choosing the hurdle arm. The situation was different for Group RR; even if both rewards were enhanced at the hurdle arm, they could be obtained more easily by going to the easy arm.

The fact that Group RR avoided the hurdle side shows that the specific effect of the hurdle was to enhance the reward rather than to increase the attractiveness of the difficult task. The effectiveness of the correlated reinforcement procedure under free-choice conditions is shown by the increasing preference for the hurdle side by the CR groups during

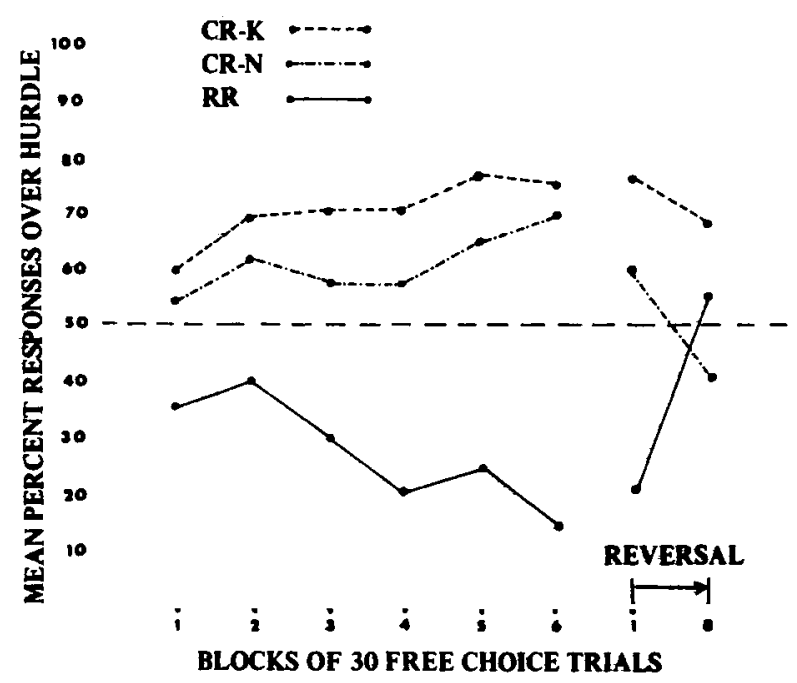

Fig. 1. Mean percentage of total responses to the difficult (hurdle) side on free-choice trials. 


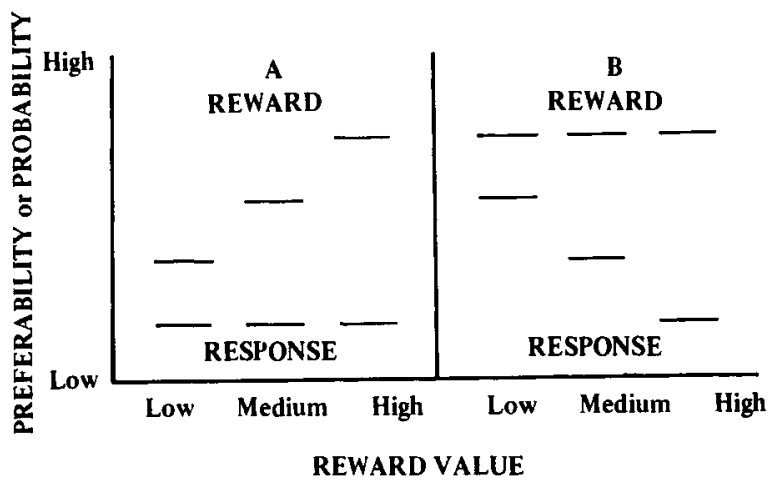

Fig. 2. Reward value as a function of the disparity between preferability of the required response and the reward. (A) The same response with differing rewards. (B) Different responses with the same reward.

training and the RR group during reversal. The persistence of the $C R$ Ss in preferring the hurdle after reward conditions were changed suggests that the hurdle may have eventually acquired strong cue or secondary reinforcement properties from its association with the preferred reward. An additional factor may be that the previously enhanced reward is available at the hurdle side $50 \%$ of the time during reversal (RR) training. The anticipated result was for the CR group to show a lesser preference for the easy side than the control group. However, the preference of the. CR Ss for the hurdle side indicates that the enhanced reward can overcome the initial undesirability of the task. The effects of effort on reward value in this situation are more striking than those reported in previous studies.

This finding, that reward value is a function of the difficulty of the task. may be interpreted in terms of Premack's (1963, 1965 ) theory of the relational nature of reinforcement. One statement of this theory would be that rewarding a given act, e.g., a barpress, by permitting a highly preferred (or probable) response. e.g.. eating, is more reinforcing than permitting a relatively less preferred response, e.g., running a wheel, (Premack, 1965, p. 151). A more general formulation is that the value of a reinforcement is a direct function of the disparity between preferability of the on-going (required) response and that of the rewarding response. This formulation is summarized in Fig. 2. The situation with the same response and different rewards is shown at the left, while the case of the same reward for different responses is shown at the right. For a given response a more preferred reward will have greater effectiveness, while for a given reward the less preferred response will be more strongly reinforced. Support for this point is given by Schaeffer $(1965$, p. 423) who found that less probable responses were relatively more reinforced (showed a proportionally greater increase in rate) than more probable responses. In the present study, response value was manipulated by presence or absence of the hurdle. The consummatory response (with one type of food) after the hurdle response was more effectively rewarding than the same response (with a discriminably different food) after the easy response.

The cognitive dissonance theory of Lawrence \& Festinger (1962) and the frustration theory of Amsel (1958) also deal with experiments of this type. These theories would involve extra attractions or anticipatory goal responses which become attached to the barrier and reward cues. However, both theories would predict that the RR group prefer the hurdle side as did the CR groups. This was clearly not the case. Furthermore, it seems unlikely that either theory could satisfactorily explain the CR groups' increasing preference for the difficult response over free-choice trials or the control group developing a preference for the hurdle side on freechoice trials when the only change was from random (RR) to correlated (CR) reinforcement.

\section{REFERENCES}

AMSEL, A. The role of frustrative nonreward in noncontinuous reward situations. Psychological Bulletin, 1958, 55, 102-119.

ARONSON, E. The effect of effort on the attractiveness of rewarded and unrewarded stimuli. Journal of Abnormal \& Social Psychology, 1961, 63, 375-380.

CHUNG, S. Effects of effort on response rate. Journal of the Experimental Analysis of Behavior, 1965, 8, 1-7.

HULL, C. L. Principles of behavior. New York: Appleton-Century, 1943.

LAWRENCE, D. H., \& FESTINGER, L. Deterrents and reinforcements. Stanford: Stanford University Press, 1962.

LEWIS, M. Some nondecremental effects of effort. Journal of Comparative \& Physiological Psychology, 1964, 57, 367-372.

MAATSCH, J. L., ADELMAN, M., \& DENNY, M. R. Effort and resistance to extinction of the barpressing response. Journal of Comparative \& Phy siological Psychology, 1954, 47, 47-50.

MOWRER, O. H., \& JONES, H. M. Extinction and behavior variability as functions of effortfulness of task. Journal of Experimental Psychology, 1943, 33, 369-386.

PREMACK, D. Prediction of the comparative reinforcement values of running and drinking. Science, 1963, 139, 1062-1063.

PREMACK, D. Reinforcement theory. In D. Levine (Ed.), Nebraska symposium on motivation. Lincoln: University of Nebraska Press, 1965. Pp. 123-180.

SCHAEFFER, R. W. The reinforcement relation as a function of instrumental response base rate. Journal of Experimental Psychology, $1965,69,419-425$.

SIEGEL, S. Nonparametric statistics for the behavioral sciences. New York: McGraw-Hill, 1956.

STACHNIK, T. The role of response effort in extinction: Much ado about nothing. Psychonomic Science, 1967, 9, 517-518.

NOTES

1. This study was supported by NIMH Grant 10532 and NIH Grant GM 13031. A portion of this material was presented at the 1965 Eastern Psychological Association Meeting.

2. Now at Williams College, Williamstown, Massachusetts. 